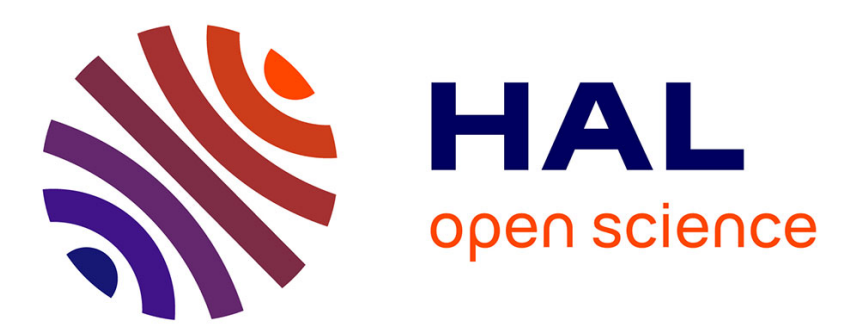

\title{
Inter-layer thermal contact resistance evolution with the degree of intimate contact in the processing of thermoplastic composite laminates
}

Arthur Lévy, Dirk Heider, John Tierney, John Gillespie

\section{- To cite this version:}

Arthur Lévy, Dirk Heider, John Tierney, John Gillespie. Inter-layer thermal contact resistance evolution with the degree of intimate contact in the processing of thermoplastic composite laminates. Journal of Composite Materials, 2014, 48 (4), pp.491-503. 10.1177/0021998313476318 . hal-03191099

\section{HAL Id: hal-03191099 \\ https://hal.science/hal-03191099}

Submitted on 6 Apr 2021

HAL is a multi-disciplinary open access archive for the deposit and dissemination of scientific research documents, whether they are published or not. The documents may come from teaching and research institutions in France or abroad, or from public or private research centers.
L'archive ouverte pluridisciplinaire HAL, est destinée au dépôt et à la diffusion de documents scientifiques de niveau recherche, publiés ou non, émanant des établissements d'enseignement et de recherche français ou étrangers, des laboratoires publics ou privés. 


\title{
Inter-layer Thermal Contact Resistance Evolution with the Degree of Intimate Contact in the Processing of Thermoplastic Composite Laminates.
}

${ }_{5} \quad$ Arthur Levy, Dirk Heider, John Tierney, John Gillespie Center for Composite Material - University of Delaware, Newark DE, USA, 19716. (302-831-8520, fax:302-831-8525, levy@udel.edu).

December 21, 2012

\begin{abstract}
This paper focuses on the contact between layers in forming processes of composite laminate. The link between the degree of intimate contact and the consequent thermal contact resistance between layers is investigated. A hot plate forming process experiment allows to propose a relation and determine the missing parameter for APC2 thermoplastic prepreg composite. Beside the new proposed relation, this work showed that the internal thermal contact resistances in the laminate is significant. Therefore, thermal modeling of forming processes of composite laminate (such as automatic tape placement) should account for this phenomenon.

keywords: Forming process, consolidation, thermal modeling, bonding mechanism, through thickness properties, on-line quality control, Carbon PEEK APC2.
\end{abstract}

\section{INTRODUCTION}

For some decades now, composite materials tend to replace traditional metallic structures in the industry. Their specific properties make them very com- 
petitive compared to metals. Although thermoset matrix composites are 25 traditionally used in the industry, for several years now, thermoplastic composites open new prospects.

Indeed, the ability to melt the matrix and the non-necessity to cure it open the way to new forming processes. One may give as an example the hot press forming [17, 21], automatic prepreg tape placement [11, 26, 23, 29, 15], or new welding techniques $[1,19]$. All those processes consist in heating the part to melt the matrix and apply pressure to bond the layers together.

In order to better monitor such processes, a good understanding of the physical phenomena involved in bonding is required. In addition to experimental campaign, numerical simulation appears to be a great tool to determine the optimal range for the process parameters. One of the very influent parameter in the process of thermoplastic is the temperature. It underlines the need for an accurate thermal modeling when simulating those processes.

Beside the temperature field, a typical objective in the simulation of composite laminate processing is to predict the quality of the bonding between the layers. With this aim, the first step is to qualify the contact between the layers at the microscopic scale. Lee and Springer [17] define a variable called degree of intimate contact that reaches 1 when contact is perfect. They propose a model of the layer surface roughness as a succession of rectangular asperities and give the degree of intimate contact as a function of temperature

Because modeling the surface as a succession of rectangular asperities is a very basic approach Yang and Pitchumani [30] proposed an improvement of the geometrical description. After reviewing the different existing approaches for modeling the degree of intimate contact evolution, we make use of the 50 Lee and Springer model that was widely used in the literature $[21,26,1,23$, $16,29,15]$.

The presents work aims at showing that the thermal properties of the laminate is highly dependent on this degree of intimate contact. Indeed, as suggested by Barasinski et al. [5, 4], a badly bonded laminate will result in air gaps between the layers and internal thermal contact resistance. This thermal contact resistance phenomenon is observed in other processes involving bad contact. For instance, in the injection molding process, the contact between the part and the mold is not perfect, and gives rise to such a thermal contact resistance $[6,22]$.

60 The thermal contact resistance at a metal interface has been studied in the past. Thomas and Probert [28] or Sridhar and Narh [27] used a 


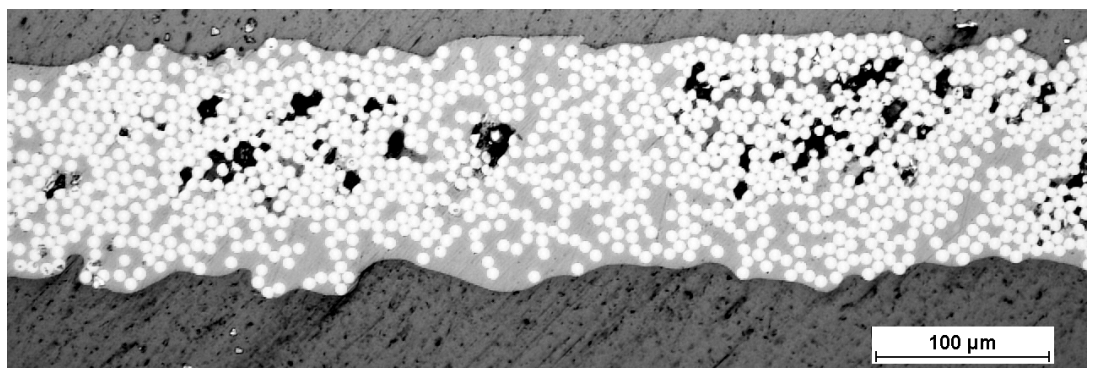

Figure 1: Micrograph of a raw APC2 tape.

modeling of the surface geometry as a succession of asperities. Nonetheless no author related the thermal contact resistance to the degree of intimate contact, especially when they are evolving, which is likely the case during the processing of a composite laminate. This paper aims at relating internal thermal contact resistance and degree of intimate contact between the layers of a laminate.

In the first part of this paper, the existing models for the degree of intimate contact are reviewed. Then, focusing on the interface, homogenization consideration allows to identify two bounds for the thermal contact resistance as a function of the degree of intimate contact. In the second part, the experimental setup is presented. It consists in a specific hot plate processing, a nanoflash apparatus for measuring thermal properties, and an optical analysis of cross sections of the laminate for measuring degree of intimate contact. The results are finally presented and discussed in section 4 and 5 . Based on experimental results, an empirical relation between thermal contact resistance and degree of intimate contact is proposed and the importance of the phenomenon is highlighted.

\section{ANALYTICAL MODEL}

\subsection{Intimate contact models}

When processing prepreg composite using tape such as the one shown on figure 1, the quality of the contact between the tapes will evolve as the surface asperities get squeezed. During the last couple of decades, several models have been proposed to predict the evolution of this contact. This first section aims at reviewing those different existing models. 
Because of the unidirectional fiber orientation, the roughness of the surface is suppose to be an extrusion in the direction of the fibers. The raw tape micrography 1 shows surface asperities of few tens of microns containing fibers. It corroborates this assumption. Therefore, the study is usually 90 restricted to two dimensions with plain strain assumption. While processing, temperature and pressure applied on the tape will allow a squeezing of the asperities. Therefore, the degree of intimate contact, defined as the contact area ratio by Lee and Springer [17] will evolve. Depending on the description of the initial surface roughness, different models have been proposed.

Rectangle model. Lee and Springer [17] modeled the initial tape roughness as a succession of rectangles as shown on Fig. 2a. The degree of intimate contact is classically defined as:

$$
D_{i c}=\frac{b}{b_{0}+w_{0}}
$$

where the geometric parameters are given on Figure 2a. The material being incompressible, the volume of the squeezed rectangles remains constant and writes:

$$
V_{0}=a_{0} b_{0}=a b,
$$

which allows to express the degree of intimate contact as a function of $a$ only:

$$
D_{i c}=\frac{a_{0} / a}{1+w_{0} / b_{0}} .
$$

Then, Lee and Springer consider a Newtonian flow of the rectangles in a lubrication framework. It is assumed that the asperity consists of an homogeneous material that behaves as a Newtonian fluid of equivalent viscosity 105 $\mu(T)$. The micrography 1 shows, indeed, that the asperities contain many fibers. At this point, improvements of the Lee and Springer model could be proposed to account for non-Newtonian behavior of the resin or better handling of the heterogeneous behavior. The following Arrhenius law

$$
\mu(T)=A \times \exp \left(\frac{E_{a}}{T[K]}\right)
$$

is proposed for the Newtonian viscosity thermal dependency, with the pre110 exponential factor $A$ and the free energy $E_{a}$ :

$$
\begin{aligned}
A & =1.14 \times 10^{-12} \text { Pa.s } \\
E_{a} & =26300 \mathrm{~K}^{-1}
\end{aligned}
$$




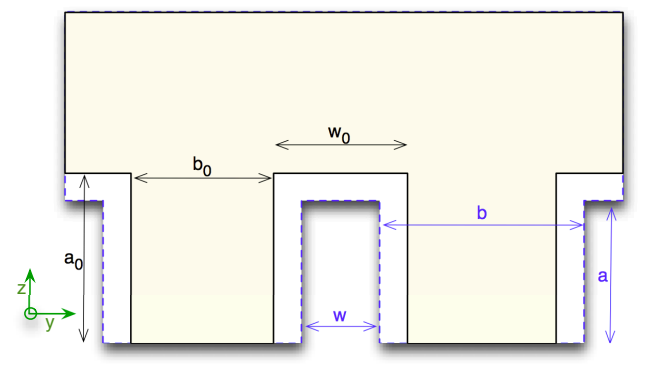

(a) As a succession of rectangles [17].

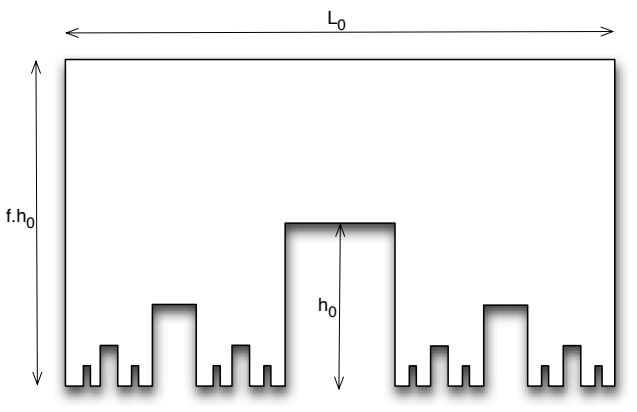

(b) As a fractal Cantor set [30].

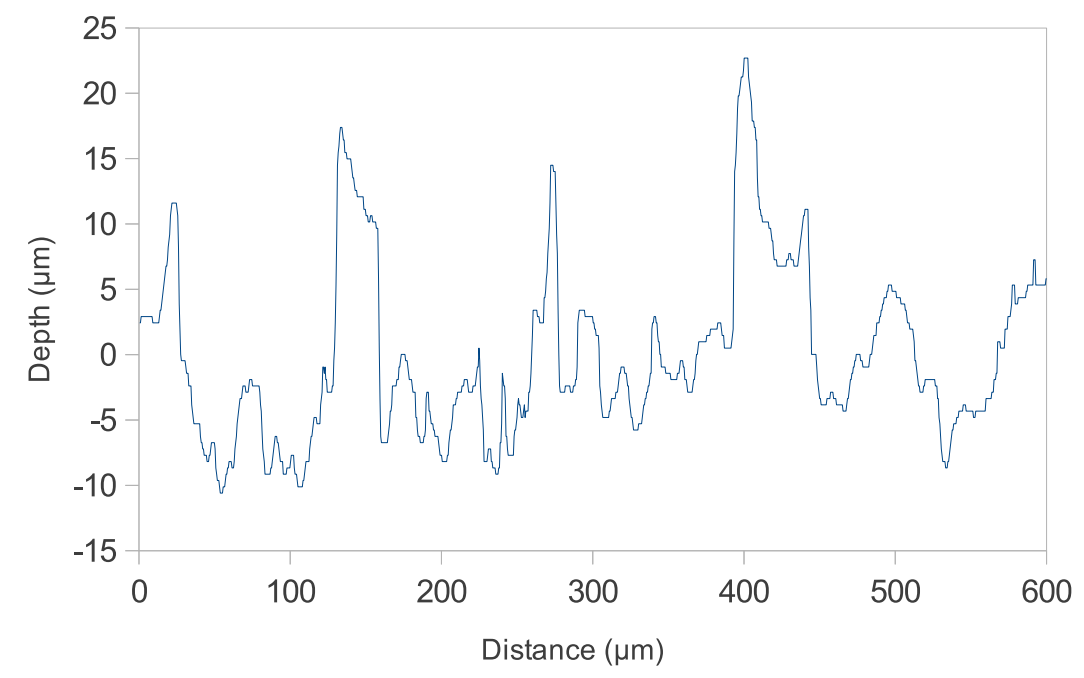

(c) Using a real measured profile.

Figure 2: Modeling of the initial surface roughness. 
The rectangle height $a$ is found to be governed by an ordinary differential equation involving the applied pressure $P_{a p p}$ and the temperature $T$. Under constant pressure and isothermal conditions, an analytical solution is obtained. Using relation (3), the model for $D_{i c}$ then writes [17]:

$$
D_{i c}=\frac{1}{1+\frac{w_{0}}{b_{0}}} \times\left[1+\frac{5 P_{a p p}}{\mu(T)}\left(1+\frac{w_{0}}{b_{0}}\right)\left(\frac{a_{0}}{b_{0}}\right)^{2} t\right]^{\frac{1}{5}} .
$$

Mantell and Springer [21] extended this model to account for non-isothermal conditions few years later.

Fractal model. Because describing the surface as a succession of rectangles is not realistic, Yang and Pitchumani [30] proposed to improve the Lee and Springer model. Indeed, the initial rectangles dimensions $a_{0}, b_{0}$ and $w_{0}$ cannot be determined physically and must therefore be fitted using experimental results. Their approach consists in describing the surface morphology as a Cantor set fractal surface (cf. fig 2b), where each geometric parameter can be obtained from a surface profile measurement. To model the squeezing of this morphology and the evolution of the degree of intimate contact Yang and Pitchumani propose the following assumption. The highest order generation of rectangle is squeezed first without any deformation of the lower generation (bigger rectangles). Once this n-th generation troughs is filled, squeezing of the next generation $n-1$ starts, and so on. The degree of intimate contact finally reaches 1 when the biggest troughs of the first generation are filled. For each successive generation $n$ the squeezing of the rectangle is modeled using a similar method as that of Lee and Springer.

Finite element model. A model using an even more realistic description of the initial surface has been lately proposed [12]. The real surface geometry measured with a profilometer, such as the one shown on figure $2 \mathrm{c}$, is meshed. (polyflow software). The authors showed a good correlation of this new model with the Lee and Springer rectangle model.

Finally, different model exist to describe the initial geometry of the tape surface. One should notice that all of these models consider the degree of intimate contact $D_{i c}$ as an accurate scalar to describe the contact at the interface. All these models also consider a Newtonian flow of the asperities and allow to relate the degree of intimate contact with the processing parameters 
(applied pressure, temperature). Further improvements of these model could account for a better description of the asperities behavior.

In this paper, for the sake of simplicity, we use the rectangle model, that has been largely used $[21,26,1,23,16,29]$, and proved its efficiency. The model chosen for $D_{i c}$ has no influence on the results shown. The presented review of the existing models for describing the evolution of the degree of intimate contact provides guideline for the reader. In this work, the degree of intimate contact is kept as a good candidate to describe the contact at the interface. Thus, this study aims at relating the thermal contact resistance and the degree of intimate contact regardless of the way the latter is obtained.

\subsection{Microscopic modeling of the thermal contact re- sistance}

The bad contact between the layers during the processing of a laminate results in an internal thermal contact resistance. This section aims at linking the degree of intimate contact $D_{i c}$ described in section 2.1 and the thermal contact resistance between layers.

We remind that the thermal contact resistance $R_{c}$ is defined as:

$$
\Phi_{z}=\frac{1}{R_{c}}\left(T^{+}-T^{-}\right)
$$

where $\Phi_{z}$ is the heat flux across the contacting interface, and $T^{+}$and $T^{-}$are the temperature on each side of the interface. Some author [28] instead define the thermal contact conductance $C_{c}$ as the inverse of the thermal contact resistance. The thermal contact resistance is linked to the morphology of the contact at the microscopic scale [28, 24].

\subsubsection{Thin Layer Model}

Let us consider the surface asperities of a tape as a thin resistive layer of thickness $a$, (as in figure 3) . This thin layer is composed of two phases: a composite phase and an air phase. The degree of intimate contact is assumed to be tractable to the whole thin layer thickness $a$, so that the volume fraction

170 of the composite phase is considered to be $D_{i c}$ in the thin layer. The two dimensional framework adopted in the intimate contact modeling is retained here. 


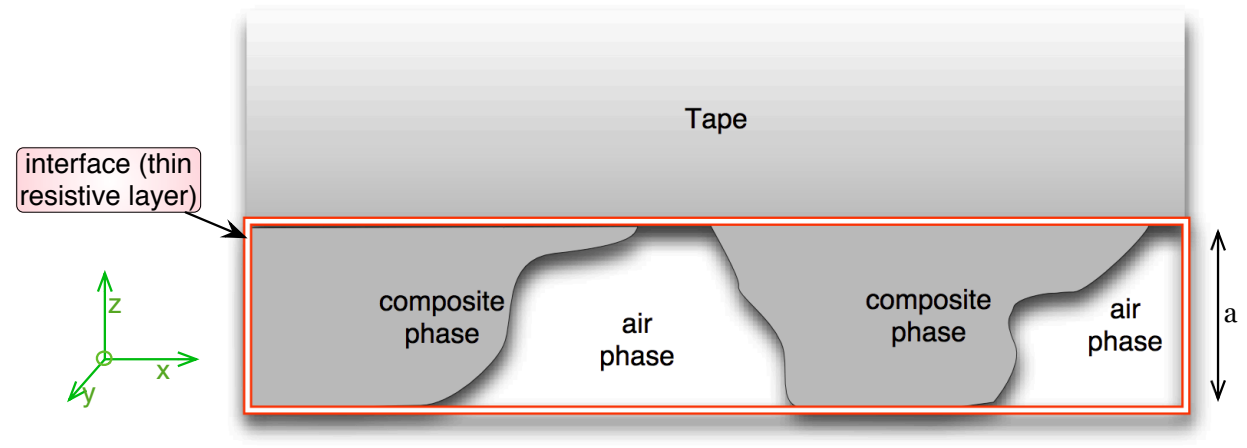

Figure 3: Representation of the interface as an heterogeneous two media thin layer.

Characterizing the thin layer properties is a spatial homogenization problem in heterogeneous media. The homogenized thermal conductivity $\boldsymbol{\lambda}^{*}$ of the thin layer is defined such that the average flux $\langle\phi\rangle$ writes

$$
\langle\phi\rangle=\boldsymbol{\lambda}^{*} \cdot\langle\nabla T\rangle \text {. }
$$

Where $\nabla T$ is the temperature gradient and $\langle\cdot\rangle$ is the spatial averaging operator in the thin layer. Let us consider the particular case where the temperature gradient is normal to the interface. For symmetry reasons, the heat flux is normal to the interface as well. The vertical direction is therefore a principal direction of $\boldsymbol{\lambda}^{*}$. This results in the following zero values in the representation of $\boldsymbol{\lambda}^{*}$ in the $(x, y, z)$ base:

$$
\boldsymbol{\lambda}^{*}=\left[\begin{array}{ccc}
\lambda_{x x}^{*} & \lambda_{x y}^{*} & 0 \\
\lambda_{x y}^{*} & \lambda_{y y}^{*} & 0 \\
0 & 0 & \lambda_{z}^{*}
\end{array}\right]_{(x, y, z)}
$$

$z$ being the direction normal to the interface.

Projecting equation (8) on this vertical direction of normal vector $\boldsymbol{e}_{z}$, one obtains:

$$
\left\langle\phi_{z}\right\rangle=\lambda_{z}^{*}\left\langle\nabla T_{z}\right\rangle
$$

Now, one wants to identify with the interface magnitudes of equation (7). We should notice that the interface is composed of two tapes in contact. Therefore there is two contacting thin layers. The interface then has a thickness $2 a$ and is comprised between $z=-a$ and $z=+a$, the origin being the 
contacting points. This assumption is enforced in the following experimental 190 setup by using a cross ply stacking that prevents asperity conformation. The average values then write:

$$
\left\langle\nabla T_{z}\right\rangle=\frac{1}{2 a} \int_{-a}^{+a} \nabla T_{z} d z=\frac{T^{+}-T^{-}}{2 a}
$$

and

$$
\left\langle\phi_{z}\right\rangle=\Phi_{z}
$$

So that:

$$
\Phi_{z}=\lambda_{z}^{*} \frac{T^{+}-T^{-}}{2 a} .
$$

Finally, using equation (7), $R_{c}$ is identified as:

$$
R_{c}=\frac{2 a}{\lambda_{z}^{*}}
$$

This equation shows that the thermal contact resistance behaves as a resistive thin layer of thickness $2 a$ and of thermal conductivity $\lambda_{z}^{*}$

In order to obtain the evolution of the thermal contact resistance $R_{c}$ with $D_{i c}$, one has to propose an homogenization law for $\lambda_{z}^{*}$. In the following paragraphs we remind the two bounds for the homogenized thermal conductivity $\lambda_{z}^{*}$ of this thin layer in this homogenization framework. Those bounds are classical in the elastic theory (for instance $[13,14]$ ) and are extended without loss of generality to the heat transfer problem. One should notice that those two bounds are valid whatever the representation of the the surface asperity is. Therefore, the following analysis stands for any degree of intimate contact model chosen among the one presented in section 2.1 .

\subsubsection{Voigt Bound}

Considering the vertical slab geometry shown on figure (4a), Beran [7] obtains the upper Voigt bound. In this case, the temperature gradient $\nabla T$ is the same in both phase and is therefore homogeneous in the thin layer

$$
\boldsymbol{\nabla} T=\frac{T^{+}-T^{-}}{2 a} \cdot \boldsymbol{e}_{z}
$$

According to the Fourier law, the respective vertical flux $\phi_{z}^{i}$ in each phase simply writes:

$$
\phi_{z}^{i}=\lambda_{z}^{i} \nabla T \cdot \boldsymbol{e}_{z}
$$




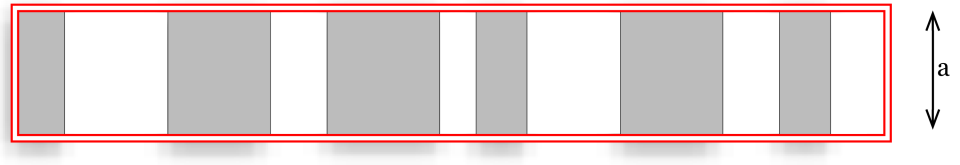

(a) Vertical slabs, homogeneous temperature gradient, Voigt bound.

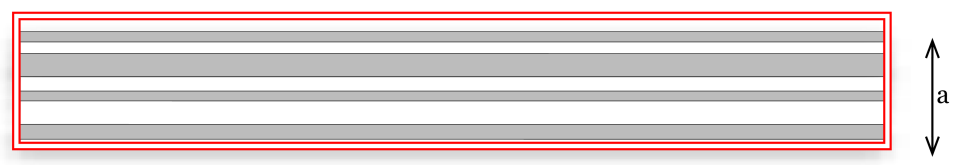

(b) Horizontal slabs, homogeneous heat flux, Reuss bound.

Figure 4: The two extreme morphologies for the thin layer leading to the two bounds.

$\lambda_{z}^{i}$ being the respective thermal conductivities of the phases in the $z$ direction. Averaging the flux gives

$$
\left\langle\phi_{z}^{i}\right\rangle=\left\langle\lambda_{z}^{i}\right\rangle \nabla T \cdot \boldsymbol{e}_{z}
$$

which allows to identify

$$
\lambda_{z}^{*}=\left\langle\lambda_{z}^{i}\right\rangle=D_{i c} \lambda+\left(1-D_{i c}\right) \lambda_{\text {air }},
$$

$\lambda_{\text {air }}$ being the conductivity of the isotropic air phase and $\lambda$ that of the composite in the transverse direction $z$. If we neglect the thermal conductivity of the air versus that of the composite, $\lambda_{z}^{*}$ finally writes

$$
\lambda_{z}^{*}=\lambda D_{i c} .
$$

Reminding that

$$
D_{i c}=\frac{\frac{a_{0}}{a}}{1+\frac{w_{0}}{b_{0}}}=D_{i c}(t=0) \frac{a_{0}}{a}
$$

we can substitute the value of $a$ in equation (14) and obtain:

$$
R_{c}=\frac{D_{i c}(t=0)}{D_{i c}^{2}} \frac{2 a_{0}}{\lambda}
$$


220

In terms of conductance, it writes:

$$
C_{c}=\frac{D_{i c}^{2} \lambda}{D_{i c}(t=0) .2 a_{0}}
$$

In this case the thermal contact conductance $C_{c}$ is proportional to the square of the degree of intimate contact. It is explained by the fact that, when $D_{i c}$ increases, both the composite phase volume fraction increases and the thickness $2 a$ of the thin layers decreases.

\subsubsection{Reuss bound}

Considering the other extreme geometry for which the interface is the stacking of alternate air and composite horizontal slabs (like one figure $4 \mathrm{~b}$ ), one obtains the lower Reuss bound [7]. In this case, the vertical flux $\phi$ is the same in both phases. The Fourier law (16) on the $z$ direction then rewrites:

$$
\phi_{z}=\lambda_{z}^{i} \nabla T_{i} \cdot e_{z}
$$

dividing by $\lambda_{z}^{i}$ and averaging, one obtains

$$
\left\langle\frac{1}{\lambda_{i}}\right\rangle \phi_{z}=\left\langle\nabla T_{i} \cdot \boldsymbol{e}_{z}\right\rangle
$$

It gives, by identifying with equation (10):

$$
\frac{1}{\lambda_{z}^{*}}=\left\langle\frac{1}{\lambda_{i}}\right\rangle
$$

or

$$
\frac{1}{\lambda_{z}^{*}}=\frac{1-D_{i c}}{\lambda_{a i r}}+\frac{D_{i c}}{\lambda}
$$

Substituting in equation (14) and using the definition of $D_{i c}$, one obtains

$$
R_{c}=D_{i c}(t=0) .2 a_{0} \times\left(\frac{1-D_{i c}}{D_{i c}} \frac{1}{\lambda_{a i r}}+\frac{1}{\lambda}\right) .
$$

\section{EXPERIMENTAL METHOD}

\subsection{Material}

The material used in this study is APC2 prepreg unidirectional composite provided by Cytec (IPS05-09-001). It is made of AS4 carbon fibers and 
PEEK thermoplastic polymer. The fiber volume content is $64 \%$. It was shipped by Cytec in 12" width tape that was cut into 3" by 3" $(76.2 \mathrm{~mm}$ by $76.2 \mathrm{~mm}$ ) squares.

The melting temperature $T_{m}$ and the glassy temperature $T_{g}$ of PEEK are known to be $[9,16]$ :

$$
\begin{aligned}
T_{m} & =334^{\circ} \mathrm{C} \\
T_{g} & =143^{\circ} \mathrm{C}
\end{aligned}
$$

The heat capacity $\rho c$ of APC2 at room temperature has been measured with a differential scanning calorimeter (DSC):

$$
\rho c=2.2 \times 10^{6} \mathrm{~J} / \mathrm{m}^{3} \mathrm{~K}
$$

245

and is in agreement with the values found in the literature $[9,2,23,18]$.

The transverse thermal conductivity $\lambda$ of the $\mathrm{APC} 2$ at room temperature can be found in the literature $[11,2,23]$ and is taken equal to:

$$
\lambda=0.63 \mathrm{~W} / \mathrm{m} \cdot \mathrm{K}
$$

\subsection{Surface Analysis}

The surface of a raw APC2 tape is analyed using cross section micrograph such as the one shown on figure 1. A simple thresholding allows to extract the profile function. The magnification is $\times 5$ and the resolution is about $2 \mathrm{pix} / \mu \mathrm{m}$. Four profiles were measured, each one on a length of about $600 \mu \mathrm{m}$. A typical profile is shown on figure 2c. The depth of the asperities $a_{0}$ is obtained as twice the standard deviation of the scanned function (similar to Yang and Pitchumani [30] approach):

$$
a_{0}=21.1 \mu \mathrm{m}
$$

\subsection{Processing}

In order to measure the thermal contact resistance between each layer of a laminate, badly bonded samples were manufactured. A specific hot plate processing was designed. 


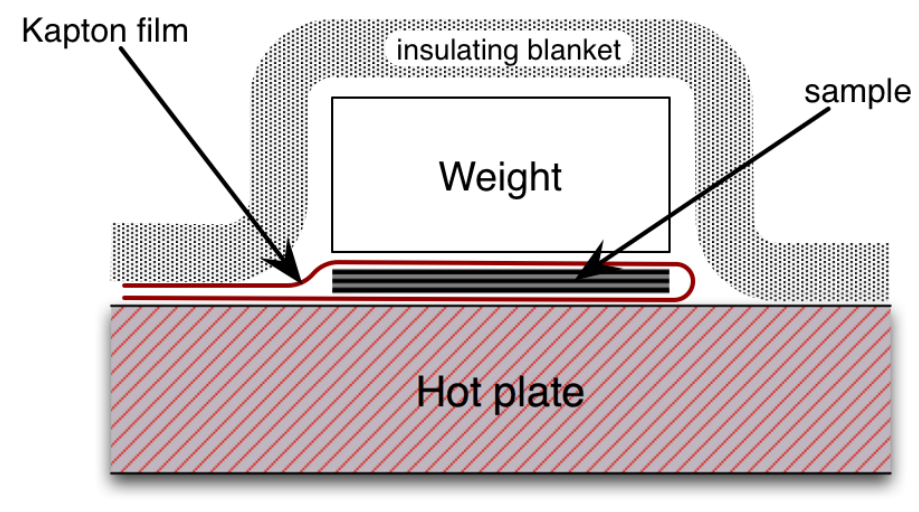

Figure 5: Experimental setup.

260

Preheating An aluminum weight of $w=793 \mathrm{~g}$ with a base of 3 in by 3 in $(76.2 \mathrm{~mm}$ by $76.2 \mathrm{~mm})$ is positioned on a hot plate and covered with a 1 in $(25.4 \mathrm{~mm})$ thick glass fiber insulating blanket. The whole setup is then heated. A K thermocouple located under the weight allows for temperature measurement at the interface between the hot plate and the weight. When reaching $400{ }^{\circ} \mathrm{C}$, we let the system stabilize for $30 \mathrm{~min}$. This ensures that the whole system composed of the hot plate and the upper aluminum weight has reached isothermal state at $400^{\circ} \mathrm{C}$. Then the sample can be processed.

Preparation Three layers of 3 in by 3 in (area $S=5806 \mathrm{~mm}^{2}$ ) of prepreg are laid up on a $0^{\circ} / 90^{\circ} / 0^{\circ}$ sequence. This allows the degree of intimate contact to be minimized by avoiding fiber conformation that would arise in a unidirectional stacking [17]. It will also help to determine the interface position accurately in the micrographs, even for perfectly bonded samples. The stacking is positioned in a folded film of Kapton coated with releasing interface (Frekote 55-NC).

Consolidation Seven different plates are processed. Each one are kept between the hot plate and the weight (see fig. 5) for different times, denoted hereunder as "consolidation times". The seven consolidation times are respectively $5 \mathrm{~s}, 10 \mathrm{~s}, 15 \mathrm{~s}, 20 \mathrm{~s}, 30 \mathrm{~s}, 1 \mathrm{~min}$ and $3 \mathrm{~min}$. During these consolidations, the weight applies a compaction pressure $P_{a p p}$. It is assumed 
homogeneous on the whole sample area, so that:

$$
P_{a p p}=\frac{w g}{S}=1340 \mathrm{~Pa}
$$

$g=9.81 \mathrm{~m} . \mathrm{s}^{-2}$ being the gravity acceleration. The three layers of thickness $e$ give a total plate thickness

$$
3 \times e=0.48 \mathrm{~mm}
$$

The composite thermal diffusivity is $\lambda /(\rho c)$. The characteristic heat diffusion time $t_{c}$ through thickness of the plates is therefore classically expressed as [8]:

$$
t_{c} \sim \frac{(3 e)^{2} \rho c}{\lambda}=0.7 \mathrm{~s} .
$$

This value is obtained using the thermal conductivity and specific heat of the composite at room temperature (equations (29) and (30)). Even if consolidation occurs at high temperature, those properties will not vary over more than an order of magnitude. Therefore, a characteristic diffusion time around $1 \mathrm{~s}$ is reasonable. During consolidation heating occurs on both sides of the sample since the hot plate and the weight were preheated. The consolidation times being way higher than $t_{c}$, the process can be considered as isothermal at temperature:

$$
T_{a p p}=400^{\circ} \mathrm{C} .
$$

\subsection{Measurement of Thermal Contact Resistance}

295 The thermal contact resistance is computed using through thickness thermal diffusivity measurements.

Nanoflash equipment (LFA 447 Nanoflash, Netzsch Instruments, Inc.) is used according to ASTM standard E1461 [3]. It consists in applying a flash of light on one side of the sample and measuring the increase in temperature on the other side using infrared measurement. The tests are performed at $25^{\circ} \mathrm{C}$. The flash light emitted with a Xenon light has a broadband between visible and near infrared. The pulse is set to "long" which correspond to a rectangular pulse of $700 \mu \mathrm{s}$. The sensor used to measure temperature on the opposite face is an InSb infrared detector cooled with liquid nitrogen. 
Sample preparation Square samples of dimension $27 \mathrm{~mm}$ by $27 \mathrm{~mm}$ were cut in the middle of the processed plate. Those samples were then coated with graphite to ensure that they are black bodies. A metal mask used in the nanoflash equipment restricts the measured area to a disk of diameter $12.7 \mathrm{~mm}$. Each sample was exposed to 10 shots at $25^{\circ} \mathrm{C}$. Through thickness diffusivity $D$ of the samples is then computed using the temperature measurement on the opposite side of the sample. The Cowan method [10], that accounts for heat loss in the air, is used. Notice that the heating resulting from the flash is lower than $0.1^{\circ} \mathrm{C}$ so that the material properties at room temperature can be used in the analysis.

5 Thermal Contact Resistance Computation Knowing the heat capacity $\rho c$ of the sample, we can obtain the equivalent through thickness thermal conductivity $\lambda_{e q}$ of each sample as:

$$
\lambda_{e q}=D \rho c
$$

The sample being modeled as a series of three layers separated by two interfaces, we can relate this equivalent conductivity to the properties of each component. Following Maillet et al. [20] work, the air gap specific heat can be neglected versus that of the composite. The contact is therefore purely resistive. Thus, considering the steady state configuration will inform us on this resistive property. The heat flux $\phi$ in each of those components would be equal and would write successively:

$$
\begin{aligned}
\phi & =\frac{\lambda}{e}\left(T_{\text {sup }}-T_{2}^{+}\right) \\
R_{c} \cdot \phi & =T_{2}^{+}-T_{2}^{-} \\
\phi & =\frac{\lambda}{e}\left(T_{2}^{-}-T_{1}^{+}\right) \\
R_{c} \cdot \phi & =T_{1}^{+}-T_{1}^{-} \\
\phi & =\frac{\lambda}{e}\left(T_{1}^{-}-T_{\text {inf }}\right)
\end{aligned}
$$

where $\lambda$ is the composite through thickness conductivity, $e$ is the tape thickness, $R_{c}$ is the thermal contact resistance searched for, and the $T_{i}$ are the temperatures defined on Figure 6 . The whole sample thickness being $3 e$, one can also write in term of equivalent conductivity:

$$
\phi=\frac{\lambda_{e q}}{3 e}\left(T_{\text {sup }}-T_{\text {inf }}\right) .
$$




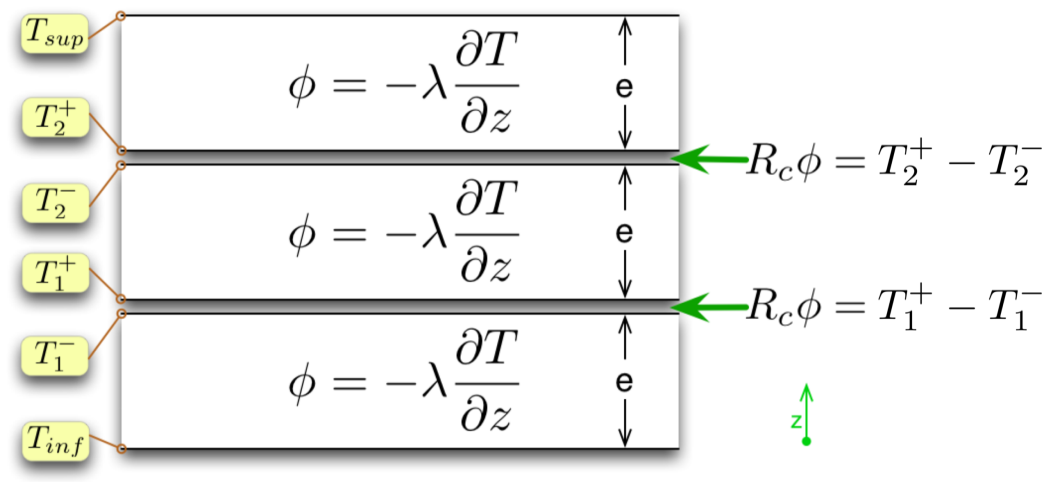

Figure 6: Unidimensional through thickness thermal modeling of the laminate.

Equations (37) and (38) finally lead to the relationship between the thermal contact resistance and the equivalent conductivity:

$$
R_{c}=\frac{3 e}{2}\left(\frac{1}{\lambda_{e q}}-\frac{1}{\lambda}\right)
$$

The results obtained for each consolidated plate are given on Figure 9.

In the presented analysis the bulk property $\lambda$ of the tape is considered constant. One may argue that the processing might change it because of internal evolution of the matter, in particular intra-layer voids content. Nonetheless, the pressure $P_{a p p}=1340 \mathrm{~Pa}$ applied is far from a real industrial pressure that might be encountered in a hot press or an autoclave. It does not likely influence the internal voids in the material. As a confirmation, the micrography corresponding to the $5 \mathrm{~s}$ and $180 \mathrm{~s}$ consolidated plates were analyzed optically and their intra-layer void content were found similar $(10.0 \%$ for the 5 s sample and $10.2 \%$ for the 180 s one). Therefore, the term consolidation, used in this paper, refers to the contact evolution between the layers, but not to void reduction that the matter would undergo in an industrial process.

One should finally notice that the intra-layer voids are distributed slightly non-homogeneously over the layer thickness (as shown on figure 7). The material conductivity $\lambda$ therefore represents the thermal conductivity of these composite tapes homogenized over the thickness. 


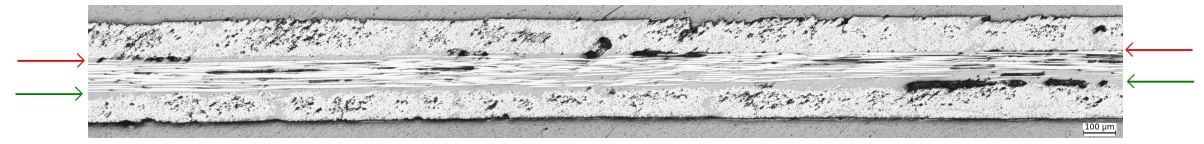

(a) General view. The arrows materialize the lines along which the intimate contact analysis is performed.

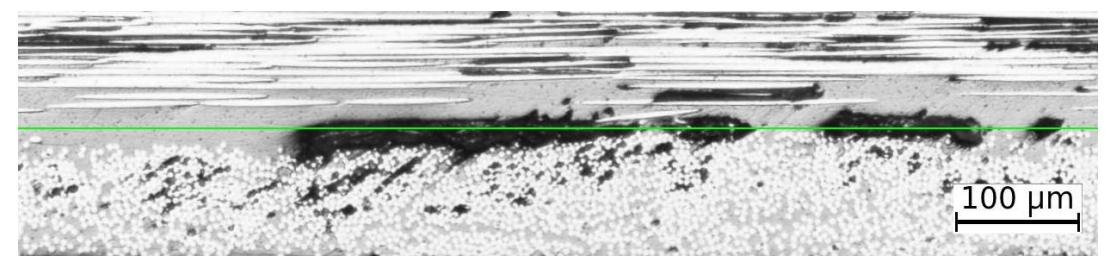

(b) Detail view with the line along which the intimate contact analysis is performed.

Figure 7: Micrograph of the $10 \mathrm{~s}$ consolidated plate (magnification $\times 5$ ).

\subsection{Measurement of Intimate Contact}

In order to relate the thermal contact resistance measured in the previous section to the intimate contact, a measurement of the degree of intimate contact $D_{i c}$ has been done on each consolidated plate.

Sample preparation Samples were cut out of the remains of each plate. The four sections facing the sample used in the nanoflash were analyzed for each plate. They were embedded in epoxy resin and polished down to $3 \mu \mathrm{m}$. Gray scale pictures such as the one shown on Figure 7 were then taken with a microscope set on a magnification of $\times 5$. The resolution is about 2 pixels per microns.

Intimate Contact Computation In order to quantify the degree of intimate contact out of those micrographs, the gray scale value was plotted along the two interfaces upper/middle and middle/lower layer. These lines are taken in the center of the interface region which is easily determined optically thanks to the fiber orientation change. The darker region are assumed to be voids whereas lighter regions can be either matrix (light gray) or fibers (white). A simple thresholding allows to obtain the contact length along these lines. The gray scale threshold value is determined independently for each picture by checking that it is in the range between the lowest gray scale value for matrix and the highest value for voids [25]. 


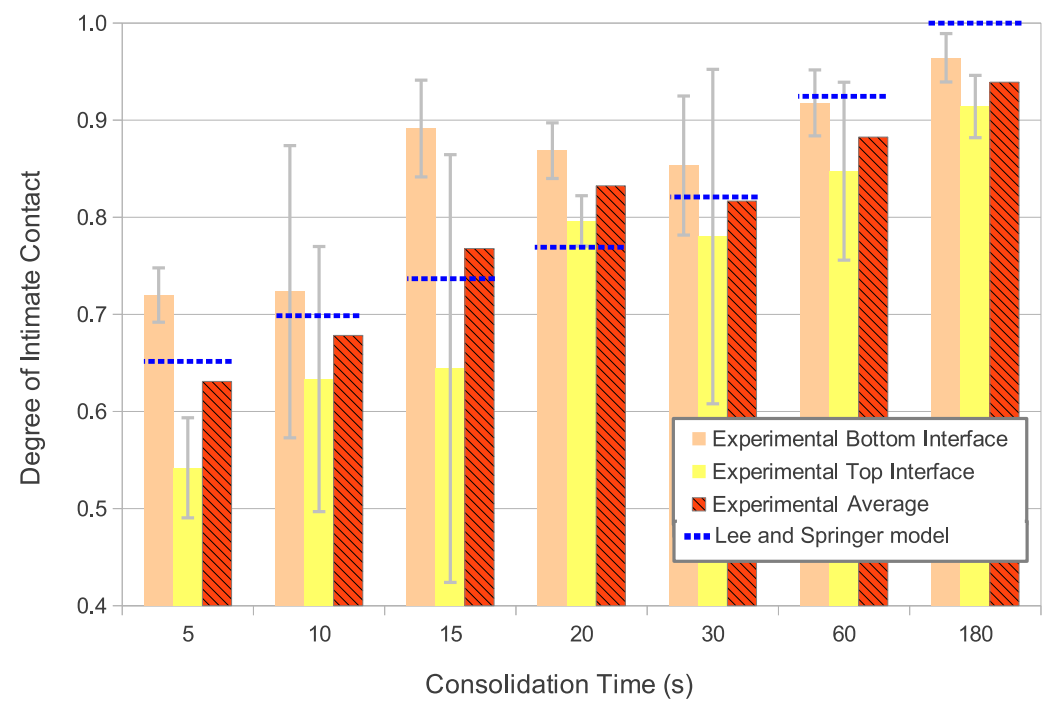

Figure 8: Measured degrees of intimate contact.

Because of the two dimensional plane strain assumption, the contact length ratio is constant in the third dimension. The degree of intimate contact is therefore directly the contact length ratio. The four values obtained for each section surrounding the nanoflash sample are then averaged successively for the upper and lower interfaces.

\section{RESULTS}

\subsection{Intimate contact}

The measured degree of intimate contact for each consolidation times are shown on Figure 8. One can notice the difference between the degree measured on the upper and lower interfaces, especially for the short time consolidation plates. This is due to the operating conditions. Their is a short lag between the positioning of the sample on the hot plate, and the closing of the mold with the hot weight. This lag (lasting usually less than a second) results in non uniformity in the heating. The heating starts earlier from the bottom face. The lower interface will reach processing temperature slightly earlier. It also explains that the longer the consolidation time, the smaller 
the difference. Hereunder the upper and lower degree of intimate contact are averaged.

385 The parameters $a_{0} / b_{0}$ and $w_{0} / b_{0}$ of the Lee and Springer model (eq. (6)) are obtained using a least square method with this average value for each plate. It consists in minimizing the norm 2 error $\operatorname{err}\left(a_{0} / b_{0}, w_{0} / b_{0}\right)$ between the model and the experiment:

$$
\operatorname{err}\left(a_{0} / b_{0}, w_{0} / b_{0}\right)=\sum_{n}\left[D_{i c}\left(a_{0} / b_{0}, w_{0} / b_{0}, t^{n}\right)-D_{i c}^{n}\right]^{2}
$$

where the summation is done over each experimental value (each consolidation time), $D_{i c}$ is the relation given in equation (6), and $D_{i c}^{n}$ are the average value of degree of intimate contact measured experimentally for each consolidation time $t^{n}$.

The minimization problem is solved numerically using a direct simplex method in Matlab (fminsearch function). One obtains

$$
\begin{aligned}
\frac{a_{0}}{b_{0}} & =1.237 \\
\frac{w_{0}}{b_{0}} & =0.743
\end{aligned}
$$

which correspond to an initial degree of intimate contact

$$
D_{i c}(t=0)=0.574
$$

These values are in agreement with the previous identification by Lee and Springer [17] who found an initial degree of intimate contact $D_{i c}=0.5$. The difference is due to difference in the material which surface roughness has evolved with the production lines in 20 years.

One should finally notice that the high dispersion of measured degree of intimate contact is related to the experimental method used. For each sample, the degrees of intimate contact were measured out of four cross sections. This corresponds to about 40 pictures for each sample. Depending on the position, the intimate contact can highly vary. As in the optical determination of void content [25], the higher the number of sections analyzed, the more accurate the degree of intimate contact measurement. Nonetheless, one should notice that Santulli et al. [25] gave a void fraction error of $10 \%$ using no more than 20 images, which suggests that our measurement is accurate within less that $10 \%$. 


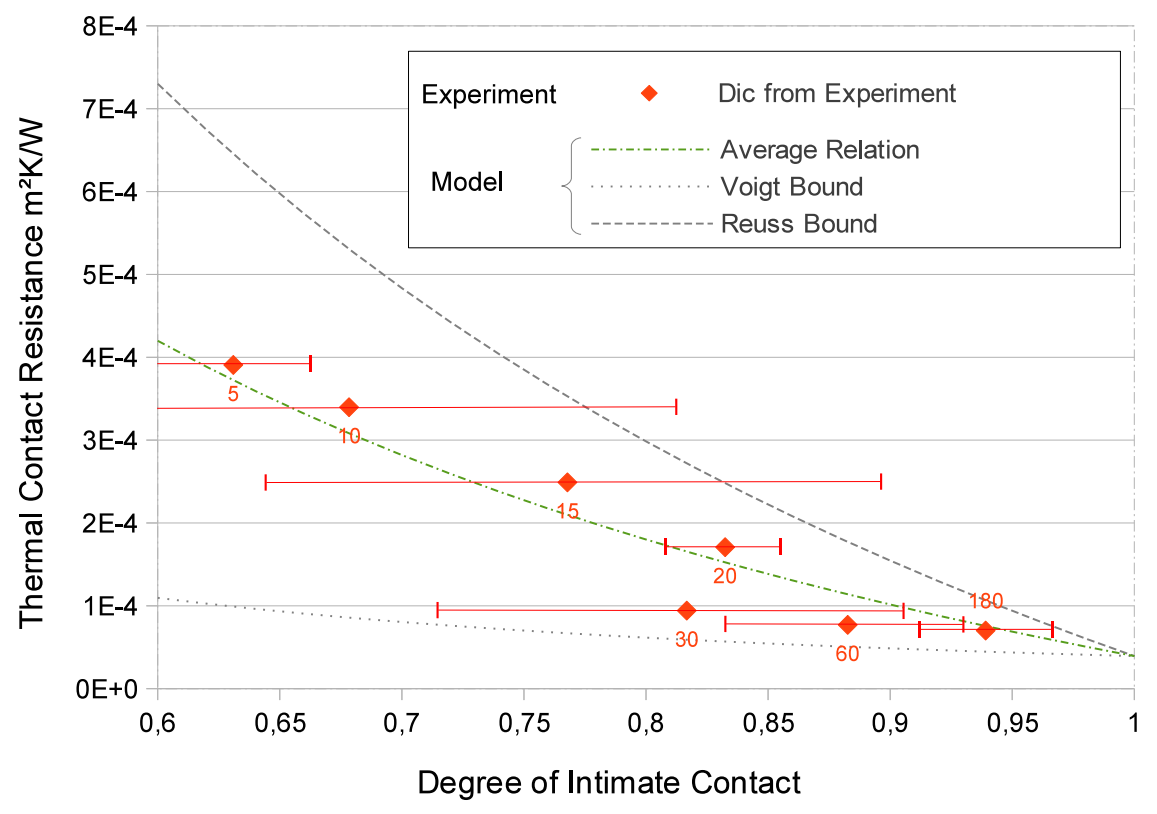

Figure 9: Thermal contact resistance vs. degree of intimate contact.

\subsection{Thermal contact resistance}

The measured thermal contact resistance are plotted on Figure 9. Once again, the relative error bars are very wide. It represents the maximum error within the 40 pictures analyzed for each point. Nevertheless, following Santulli et al. [25] approach, the error on the deduced void content is lower than $10 \%$.

The Reuss and Voigt bounds (eq. (21) and (27)) are plotted on the graph using the value of $a_{0}$ obtained in section 3.2. One can notice that the experimental values lie within the bounds.

Following the Voigt-Reuss-Hill [13] principle, one may suggests a basic arithmetic average of the Voigt and Reuss bound as an empirical relation between thermal contact resistance and degree of intimate contact:

$$
R_{c}\left(D_{i c}\right)=D_{i c}(t=0) a_{0}\left(\frac{1}{\lambda D_{i c}^{2}}+\frac{1-D_{i c}}{\lambda_{a i r} D_{i c}}+\frac{1}{\lambda}\right) \text {. }
$$

Note that this relation stands for the experimental points on figure 9 and does not depend on the intimate contact model used. 


\section{DISCUSSION}

425 In the forming process of thermoplastic composite, predicting the quality of the bonding between layers requires a good modeling of the intimate contact evolution. The presented results focused on two coupled phenomena: the intimate contact evolution and the thermal contact resistance at the interfaces. Those two properties are shown to be related.

430

\subsection{Linking Thermal Contact Resistance and Degree of Intimate Contact}

Equation (43) proposes a relation between the thermal contact resistance and the degree of intimate contact. Note that all the parameters in the relation are physical. Even $a_{0}$, the thickness of the initial thin layer, can be as described in section 3.2. This relation can be used as is, regardless of the modeling of the degree of intimate contact $D_{i c}$.

Because this relation uses only physical parameters, its temperature dependency is straightforward: $\lambda$ and $\lambda_{\text {air }}$ only need to be adapted to reflect their variation with temperature. Literature values such as Cogswell [9] or Ageorges et al. [2] might be used. The relation can therefore be extended to high temperature and used for in situ modeling of thermal contact resistance while processing.

The experimental work consisted in analyzing the produced samples by

1. The nanoflash experiment allows to quickly obtain the average thermal contact resistance over the area of the flash beam.

2. The optical analysis requires a large number of image processing to obtain an accurate spatial average value of the degree of intimate contact. Given the sample preparation requirement (embedding and polishing), this is a time-consuming measurement.

The results showed a clear link between intimate contact and thermal resistance. Therefore, in a production framework, one could consider the thermal contact resistance to be the discriminant parameter for qualifying the quality of the intimate contact. Instead of requiring a minimum degree of intimate contact, one may require a maximum thermal contact resistance per layer. 
Actually, implementation of a nanoflash device on a production site to perform in-situ control is possible, and even easier, than an ultrasonic technique because it is a non contacting method. A similar non destructive thermal investigation method was suggested by Maillet et al. [20] to predict delamination on composite part during their lifetime.

\subsection{Importance of the Phenomenon}

In a forming process, the intimate contact and heat transfer phenomena are fully coupled. The degree of intimate contact depends on the tempera-

ture history and the temperature depends on the thermal contact resistance that depends on the degree of intimate contact. Accurately modeling an industrial forming process in order to predict the degree of intimate contact therefore requires the simultaneous solving of the heat transfer and the degree of intimate contact evolution. With this aim, relation (43) should be used in conjunction with an appropriate degree of intimate contact model (as reviewed in section 2.1).

Moreover the thermal contact resistance values obtained are ranging between $5 \times 10^{-5} \mathrm{~m}^{2} \mathrm{~K} / \mathrm{w}$ and $5 \times 10^{-4} \mathrm{~m}^{2} \mathrm{~K} / \mathrm{w}$. Those values are comparable with the ones obtained in other forming processes, such as the thermal contact resistance between mold and part in injection molding [22, 6]. This confirms the importance of the phenomenon that will affect the heat transfer during processing of composite laminate.

As a matter of fact, the thermal contact resistance highly modifies the equivalent through thickness thermal conductivity. As shown on Figure 10, neglecting the phenomenon would be similar to making a mistake of more than $50 \%$ on the through thickness thermal conductivity.

Finally one should notice that the present study focused on the intimate contact, which is an interlayer void. To this end, the samples studied exhibited similar intra-layer void content. In a real industrial process, the intra-layer void content is likely to evolve too, resulting in an evolution on the bulk composite property. This issue might be addressed in future studies.

\section{CONCLUSION}

A relation between the degree of intimate contact and the internal thermal contact resistance between the layers was obtained with an experimental 


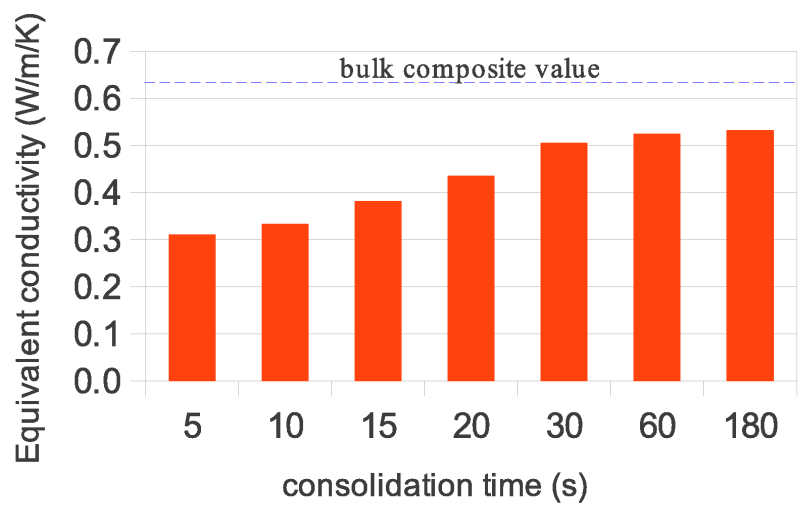

Figure 10: Equivalent thermal conductivity through thickness for different consolidation times.

campaign. The measured thermal contact resistance values for badly consolidated sample are high. It shows that the phenomenon is of first importance. Indeed, because of this internal thermal contact resistance, the equivalent through thickness thermal conductivity can almost drop by a factor 2 .

This thermal contact resistance should definitely be taken into account in the thermal simulation of composite laminate forming processes. This is the aim of the future development of a simulation tool accounting for the full coupling between intimate contact and heat transfer in the automatic tape placement process.

\section{ACKNOWLEDGEMENT}

The authors would like to thank EADS CTO North America and especially Marc Fraser for funding this research. Our thanks also go to Patrice Lefebure, from EADS IW, France who allowed this collaboration.

\section{References}

[1] C. Ageorges, L. Ye, and M. Hou. (2001). Avdances in Fusion Bonding Techniques for Joining Thermoplastic Matrix Composites: a Review. Composites Part A: applied science and manufacturing, 32(6):839-857. 
[2] C. Ageorges, L. Ye, Y.-W. Mai, and M. Hou. (1998). Characteristics of resistance welding of lap shear coupons : Part I : Heat transfer. Composites, 29A:899-909.

[3] ASTM International. (2001). ASTM E1461 - 07 Standard Test Method for Thermal Diffusivity by the Flash Method. West Conshohocken, PA. URL www. astm.org.

[4] A. Barasinski, A. Leygue, E. Soccard, and A. Poitou. (2011). In situ consolidation for thermoplastic tape placement process is not obvious. In AIP Conference Proceedings, pages 948-953.

[5] A. Barasinski, A. Leygue, E. Soccard, and A. Poitou. (2010). An Improvement in Thermal Modeling of Automated Tape Placement Process. In International Conference on Advances in Materials and Processing Technologies (AMPT2010), pages 185-190.

[6] A. Bendada, A. Derdouri, M. Lamontagne, and Y. Simard. (2004). Analysis of thermal contact resistance between polymer and mold in injection molding. Applied Thermal Engineering, 24(14-15):2029-2040.

[7] M. J. Beran. (1968). Statistical Continuum Theories. Interscience publishers, New York - London - Sydney.

[8] H. S. Carslaw and J. C. Jaeger. (1959). Conduction of Heat in Solids. Clarendon Press - Oxford.

[9] F. N. Cogswell. (1992). Thermoplastic aromatic polymer composites. Woodhead.

[10] R. D. Cowan. (1963). Pulse Method of Measuring Thermal Diffusivity at High Temperatures. Journal of Applied Physics, 34(4):926-927.

[11] S. Grove. (1988). Thermal modelling of tape laying with continuous carbon fibre-reinforced thermoplastic. Composites, 19(5):367-375.

[12] M. B. Gruber, I. Z. Lockwood, T. L. Dolan, S. B. Funck, J. Tierney, P. Simacek, J. W. J. Gillespie, S. Advani, B. J. Jensen, and R. J. Cano. (2012). Thermoplatic In Situ Placement Requires Better Impregnated Tapes and Tows. In SAMPE Conference - Baltimore. 
[13] R. Hill. (1952). The Elastic Behaviour of a Crystalline Aggregate. Proceedings of the Physical Society. Section A, 65(5):349-354.

[14] T. Kanit, S. Forest, I. Galliet, V. Mounoury, and D. Jeulin. (2003). Determination of the size of the representative volume element for random composites: statistical and numerical approach. International Journal of Solids and Structures, 40(13-14):3647-3679.

[15] M. A. Khan, P. Mitschang, and R. Schledjewski. (2010). Identification of some optimal parameters to achieve higher laminate quality through tape placement process. Advances in Polymer Technology, 29(2):98-111.

[16] J.-F. Lamethe, P. Beauchene, and L. Leger. (2005). Polymer dynamics applied to PEEK matrix composite welding. Aerospace Science and Technology, 9(3):233-240.

[17] W. I. Lee and G. S. Springer. (1987). A model of the manufacturing process of thermoplastic matrix composites. Journal of Composite Materials, 21(11):1017-1055.

[18] A. Levy, S. Le Corre, N. Chevaugeon, and A. Poitou. (2011). A levelset based approach for the finite element simulation of a forming process involving multiphysics coupling : ultrasonic welding of thermoplastic composites. European Journal of Mechanics - A/Solids, 30(4):501-509.

[19] A. Levy, S. Le Corre, A. Poitou, and E. Soccard. (2011). Ultrasonic Welding of Thermoplastic Composites, Modeling of the Process Using Time Homogenization. International Journal for Multiscale Computational Engineering, 9(1):53-72.

[20] D. Maillet, A. Houlbert, S. Didierjean, A. Lamine, and A. Degiovanni. (1993). Non-destructive thermal evaluation of delaminations in a laminate: Part I - Identification by measurement of thermal contrast. Composites Science and Technology, 47(2):137-153.

[21] S. Mantell and G. Springer. (1992). Manufacturing process models for thermoplastic composites. Journal of Composite Materials, 26(16): $2348-2377$.

[22] H. Masse, E. Arquis, D. Delaunay, S. Quilliet, and P. Le Bot. (2004). Heat transfer with mechanically driven thermal contact resistance at the 
polymer/mold interface in injection molding of polymers. International Journal of Heat and Mass Transfer, 47(8-9):2015-2027.

[23] C. Nicodeau. (2005). Modélisation du Soudage en Continu des composites à Matrice Thermoplastique. PhD thesis, Ecole Nationale Superieure d'Arts et Métiers de Paris. URL http://tel.archives-ouvertes .fr/ pastel-00001506.

575 [24] J. Salgon, F. Robbe-Valloire, J. Blouet, and J. Bransier. (1997). A mechanical and geometrical approach to thermal contact resistance. International Journal of Heat and Mass Transfer, 40(5):1121-1129.

[25] C. Santulli, R. Gil, A. Long, and M. J. Clifford. (2002). Void content measurements in commingled E-glass/polypropylene composites using image analysis from optical micrographs. Science and Engineering of Composite Materials, 10(2):77-90.

[26] F. Sonmez and H. Hahn. (1997). Analysis of the on-line consolidation process in thermoplastic composite tape placement. Journal of Thermoplastic Composite Materials, 10(6):543.

[27] L. Sridhar and K. A. Narh. (1997). Measurement and Modeling of Thermal Contact Resistance at a Plastic-Metal Interface. In ANTEC Conference, Toronto, volume -1, pages 2273-2277.

[28] T. Thomas and S. Probert. (1970). Thermal contact resistance: The directional effect and other problems. International Journal of Heat and Mass Transfer, 13(5):789-807.

[29] J. Tierney and J. W. Gillespie. (2006). Modeling of in situ strength development for the thermoplastic composite tow placement process. Journal of Composite Materials, 40(16):1487-1506.

[30] F. Yang and R. Pitchumani. (2001). A fractal Cantor set based description of interlaminar contact evolution during thermoplastic composites processing. Journal of Materials Science, 36(19):4661-4671. 